\begin{tabular}{|c|l|}
\hline Title & Improving thermal efficiency by reducing cooling losses in hydrogen combustion engines \\
\hline Author(s) & Shudo, Toshio \\
\hline Citation & $\begin{array}{l}\text { International Journal of Hydrogen Energy, 32(17), 4285-4293 } \\
\text { https://doi.org/40.1016/.ijhydene.2007.06.002 }\end{array}$ \\
\hline Issue Date & $2007-12$ \\
\hline Doc URL & http://hdl.handle.net/2115/30295 \\
\hline Type & article (author version) \\
\hline File Information & IJHE32-17.pdf \\
\hline
\end{tabular}

Instructions for use 


\title{
Improving thermal efficiency by reducing cooling losses in hydrogen combustion engines
}

Toshio Shudo

Applied Energy System Group, Division of Energy and Environmental Systems, Hokkaido University

N13 W8, Kita-Ward, Sapporo, Hokkaido, 060-8628, JAPAN

Email: shudo@eng.hokudai.ac.jp

Tel/Fax: +81-11-706-6402

\begin{abstract}
Hydrogen can be readily used in spark ignition engines as a clean alternative to fossil fuels. However the higher burning velocity and shorter quenching distance of hydrogen compared with hydrocarbons cause a larger heat transfer from the burning gas to the combustion chamber walls. Because of this cooling loss, the thermal efficiency of hydrogen-fueled engines is sometimes lower than that of conventionally fueled engines. Therefore, reducing the cooling loss is a crucial element in improving the thermal efficiency of hydrogen combustion engines. Previous research by the author and others has proposed the direct injection stratified charge as a technique for reducing the cooling loss in hydrogen combustion and shown its effect in reducing cooling loss through experiments in a constant volume combustion vessel. However, it is known that a reduction in cooling loss does not always improve thermal efficiency due to a simultaneous increase in the exhaust heat loss. This paper explains the relation between cooling loss reduction and thermal efficiency improvements by the direct injection stratified charge in hydrogen combustion engines.
\end{abstract}

Key Words: Hydrogen engine, Thermal efficiency, Cooling loss, Exhaust loss, Stratified charge, Combustion

\section{Introduction}

Hydrogen can be used as a clean alternative to petroleum fuels, and its use as a vehicle fuel is promising in the effects to establish environmentally friendly mobility systems. The polymer electrolyte fuel cell and the internal combustion engine are the two leading potential power systems for hydrogen fueled vehicles. However with fuel cell systems there are still many issues to be addressed before their extensive use in large production volumes in spite of the high theoretical efficiency. At the same time, spark ignition internal combustion engines can readily use hydrogen as a fuel with only minor modifications, making hydrogen engines a more practical technology and that is simpler to implement than current fuel cells. However, hydrogen has unique combustion characteristics influencing the thermal efficiency of engines. Particularly, hydrogen has a higher burning velocity, a shorter quenching distance, and a higher thermal conductivity than hydrocarbon fuels [1]. The high burning velocity causes a rapid flame propagation in hydrogen combustion engines resulting in an intense convection of the burning gas and a large heat transfer from the burning gas to the combustion chamber walls [2]. The short quenching distance and the high thermal conductivity of hydrogen may also increase the heat transfer. Shorter quenching distance leads to thinner temperature boundary layer on the walls, and higher thermal conductivity leads to larger heat transfer from unburned mixture to the walls. Because of the cooling loss, a heat transfer loss, the thermal efficiency of hydrogen combustion engines is sometimes lower than engines fueled with conventional hydrocarbon fuels. Figure 1 shows the maximum heat flux to the cylinder head, the cooling loss fraction and the indicated thermal efficiency for hydrogen and methane combustion at stoichiometric conditions [2]. A large cooling loss fraction for hydrogen combustion resulted in lower thermal efficiency than methane combustion. Therefore, a reduction in the cooling loss is very important for improving the thermal efficiency of hydrogen combustion engines.

It is known that direct injection stratified charge suppresses knocking compared to homogeneous charge [3]. The mechanism was attributed to a much leaner mixture in the end gas. Such presence of leaner mixture implies that burned gas adjacent to the wall will be at a lower temperature. Previous research [4] using a constant volume combustion vessel has shown that a direct injection stratified charge is an effective technique to reduce the cooling loss in hydrogen combustion. Direct fuel injection, internal mixing of fuel and air, also has the advantage that it can avoid backfire, the flame propagation from combustion chamber to the intake pipe that may stop the engine. Backfire is the major barrier restricting the power output from premixed charge type hydrogen engines [5-6]. The mixture stratification also reduces NOx emissions at near-stoichiometric overall equivalence ratios in spark ignition engine [3]. This is because Zel'dovich NO, which is the main source of NOx emission from engine combustion, decreases 
with increasing or decreasing in mixture equivalence ratio from stoichiometry. With near-stoichiometric overall equivalence ratios, the stratification reduces the fraction of near-stoichiometric mixture resulting in lower NOx emissions. Therefore, when the equivalence ratio is increased toward stoichiometry for obtaining higher power output in a hydrogen engine, stratified charge emits lower engine-out NOx emissions than homogeneous charge. Then NOx can be aftertreated with some types of catalysts to meet vehicle tail-pipe emissions standards.

Mixture stratification, in which the fuel-air mixture adjacent to the combustion chamber walls burns at lower temperatures, is an attractive option for hydrogen engine to reduce the cooling loss at relatively high engine loads. However, for conventionally fueled engines it is known that a reduction in the cooling loss does not always lead to an effective improvement in thermal efficiency because of a simultaneous increase in the exhaust heat loss. With this background, the current research analyzes the relation between cooling loss reduction and thermal efficiency improvement by a direct injection stratified charge in hydrogen combustion engines.

\section{Analysis}

\subsection{Thermal efficiency factors}

The indicated thermal efficiency $\eta_{\mathrm{i}}$ of a spark ignition engine can be described with the theoretical thermal efficiency of the Otto cycle $\eta_{\mathrm{th}}$ and the diagram factor $\eta_{\mathrm{g}}[7]$.

$$
\eta_{\mathrm{i}}=\eta_{\mathrm{th}} \eta_{\mathrm{g}}
$$

The diagram factor $\eta_{\mathrm{g}}$ is expressed with the degree of constant volume $\eta_{\mathrm{gh}}$, the combustion efficiency $\eta_{\mathrm{u}}$, and the cooling loss fraction $\phi_{\mathrm{w}}$ as

$$
\eta_{\mathrm{g}}=\eta_{\mathrm{glh}} \eta_{\mathrm{u}}\left(1-\phi_{\mathrm{w}}\right)
$$

Therefore, the indicated thermal efficiency can be expressed as

$$
\eta_{\mathrm{i}}=\eta_{\mathrm{th}} \eta_{\mathrm{glh}} \eta_{\mathrm{u}}\left(1-\phi_{\mathrm{w}}\right)
$$

The theoretical thermal efficiency of the Otto cycle $\eta_{\text {th }}$ is defined with the following equation using the stroke volume $\mathrm{V}_{\mathrm{h}}$, the clearance volume $\mathrm{V}_{\mathrm{c}}$, and the specific heat ratio of working fluid $\gamma$, as

$$
\eta_{\mathrm{th}}=1-\left(\left(\mathrm{V}_{\mathrm{h}}+\mathrm{V}_{\mathrm{c}}\right) / \mathrm{V}_{\mathrm{c}}\right)^{1-\gamma}
$$

Because the theoretical thermal efficiency $\eta_{\mathrm{th}}$ is determined by the geometrical dimensions of the engine and the specific heat ratio of the working fluid, changes in the indicated thermal efficiency $\eta_{\mathrm{i}}$ of an engine are determined mainly by the diagram factor $\eta_{\mathrm{g}}$.

\subsection{Degree of constant volume}

The constant volume combustion at top dead center is the heat input closest to that of the ideal cycle by Otto. The degree of constant volume $\eta_{\mathrm{glh}}$, a component of the diagram factor $\eta_{\mathrm{g}}$, is defined as follows [2].

$$
\eta_{\mathrm{glh}}=1 /\left(\eta_{\mathrm{th}} Q\right) \int\left(1-\left(\left(\mathrm{V}_{\mathrm{h}}+\mathrm{V}_{\mathrm{c}}\right) / V\right)^{1-\gamma}\right) \mathrm{d} Q / \mathrm{d} \theta \mathrm{d} \theta
$$

here, $\theta$ is the crank angle, $V$ is the cylinder volume as a function of the crank angle, $\mathrm{d} Q / \mathrm{d} \theta$ is the apparent rate of heat release, and $Q$ is the cumulative apparent heat release. This research calculated the apparent rate of heat release $\mathrm{d} Q / \mathrm{d} \theta$ from the measured pressure diagram using the following equation [8].

$$
\mathrm{d} Q / \mathrm{d} \theta=V /(\gamma-1) \mathrm{d} P / \mathrm{d} \theta+\gamma P /(\gamma-1) \mathrm{d} V / \mathrm{d} \theta-P V /(\gamma-1)^{2} \mathrm{~d} \gamma / \mathrm{d} \theta
$$

The apparent rate of heat release $\mathrm{d} Q / \mathrm{d} \theta$ calculated with the above equation was integrated by the crank angle to the valve opening timing to obtain the cumulative apparent heat release $Q$.

\subsection{Cooling loss fraction}

A further component of the diagram factor $\eta_{\mathrm{g}}, \eta_{\mathrm{u}}\left(1-\phi_{\mathrm{w}}\right)$, can be obtained from the cumulative apparent heat release $Q$ and the fuel heat $Q_{\text {fuel }}$ supplied in a cycle [2]. The cumulative apparent heat release $Q$ calculated from the measured pressure data is influenced by the heat transfer from the burning gas to the combustion chamber walls, the apparent heat release fraction $Q / Q_{\text {fuel }}$ can be expressed by the following equation with the cumulative real heat release $Q_{\mathrm{B}}$ and the cumulative cooling loss heat $Q_{\mathrm{C}}$.

$$
Q / Q_{\text {fuel }}=\left(Q_{\mathrm{B}}-Q_{\mathrm{C}}\right) / Q_{\text {fuel }}
$$

The product of the supplied fuel heat $Q_{\text {fuel }}$ and the combustion efficiency $\eta_{\mathrm{u}}$ gives the cumulative real heat release $Q_{\mathrm{B}}$, and the apparent heat release fraction is rewritten as

$$
Q / Q_{\text {fuel }}=\eta_{\mathrm{u}}\left(Q_{\mathrm{B}}-Q_{\mathrm{C}}\right) / Q_{\mathrm{B}}
$$


By defining the cooling loss fraction $\phi_{\mathrm{v}}$ as the fraction of the cumulative cooling loss heat $Q_{\mathrm{C}}$ to the cumulative real heat release $Q_{\mathrm{B}}$, the apparent heat release fraction $Q / Q_{\text {fuel }}$ corresponds to a function of the combustion efficiency and the cooling loss fraction $\eta_{\mathrm{u}}\left(1-\phi_{\mathrm{w}}\right)[2]$.

$$
Q / Q_{\text {fuel }}=\eta_{\mathrm{u}}\left(1-\phi_{\mathrm{w}}\right)
$$

From this equation the cooling loss fraction $\phi_{\mathrm{w}}$ can be written as

$$
\phi_{\mathrm{w}}=1-Q /\left(\eta_{\mathrm{u}} Q_{\text {fuel }}\right)
$$

Here, the cooling loss fraction $\phi_{\mathrm{w}}$ was calculated using Equation (10) with the combustion efficiency obtained from the measured oxygen concentration in the exhaust gas.

\subsection{Exhaust loss fraction}

Exhaust heat loss $Q_{\text {ex }}$, the heat carried away by the exhaust gas per cycle, is described with the supplied heat to the cycle $Q$ and the indicated work $W_{\mathrm{i}}$.

$$
\underset{\text { (11) }}{Q_{\mathrm{ex}}}=Q-W_{\mathrm{i}}
$$

From Equation (9) the cumulative apparent heat release $Q$ can be written by the following equation.

$$
Q=\eta_{\mathrm{u}}\left(1-\phi_{\mathrm{w}}\right) Q_{\text {fuel }}
$$

The definition of the indicated thermal efficiency $\eta_{\mathrm{i}}$ and Equation (1) give the following relation.

$$
W_{\mathrm{i}}=\eta_{\mathrm{th}} \eta_{\mathrm{glh}} \eta_{\mathrm{u}}\left(1-\phi_{\mathrm{w}}\right) Q_{\text {fuel }}
$$

From Equations (12) and (13) the indicated work $W_{\mathrm{i}}$ can be described as

$$
W_{\mathrm{i}}=\eta_{\mathrm{th}} \eta_{\mathrm{glh}} Q
$$

(14)

Then from Equations (11), (12), and (14), the exhaust heat loss $Q_{\mathrm{ex}}$ is described as

$$
Q_{\mathrm{ex}}=\left(1-\eta_{\mathrm{th}} \eta_{\mathrm{glh}}\right) \eta_{\mathrm{u}}\left(1-\phi_{\mathrm{w}}\right) Q_{\text {fuel }}
$$

This paper defines the exhaust loss fraction $\phi_{\mathrm{ex}}$ as the fraction of exhaust gas heat $Q_{\mathrm{ex}}$ to the supplied fuel heat $Q_{\text {fuel }}$

$$
\phi_{\mathrm{ex}}=Q_{\mathrm{ex}} / Q_{\text {fuel }}
$$

From Equations (15) and (16),

$$
\phi_{\mathrm{ex}}=\left(1-\eta_{\mathrm{th}} \eta_{\mathrm{glh}}\right) \eta_{\mathrm{u}}\left(1-\phi_{\mathrm{w}}\right)
$$

By using all of the above thermal efficiency factors, the paper analyzes the relations among them and their influences on the thermal efficiency in hydrogen combustion engines.

\section{Results and Discussions}

\subsection{Relation between cooling loss fraction and other efficiency factors}

Figure 2 shows the calculated influence of the cooling loss fraction $\phi_{\mathrm{w}}$ on the indicated thermal efficiency $\eta_{\mathrm{i}}$, exhaust loss fraction $\phi_{\mathrm{ex}}$, and the ratio of the two $\eta_{\mathrm{i}} / \phi_{\mathrm{ex}}$ with the parameter of the degree of constant volume $\eta_{\mathrm{glh}}$. The calculations employed the compression ratio $\varepsilon$ of 8.5 , the specific heat ratio of the working fluid $\gamma=1.4$, and the combustion efficiency $\eta_{\mathrm{u}}=0.98$. Unlike hydrocarbon fuels, the specific heat ratio of hydrogen is very close to that of air, and this provide a slight advantage of hydrogen engines over conventionally fueled ones. Both the indicated thermal efficiency $\eta_{\mathrm{i}}$ and exhaust loss fraction $\phi_{\mathrm{ex}}$ increase with decreasing cooling loss fraction $\phi_{\mathrm{w}}$, at a degree of constant volume $\eta_{\mathrm{glh}}$. Here it must be noted that regardless of the cooling loss fraction $\phi_{\mathrm{w}}$, the ratio $\eta_{\mathrm{i}} / \phi_{\mathrm{ex}}$ is constant for a degree of constant volume $\eta_{\mathrm{glh}}$. This means that the indicated thermal efficiency and the exhaust loss fraction are improved at the same rate when the cooling loss is reduced, if the degree of constant volume remains constant. This is also shown by Equation (18), which is derived from Equations (3) and (17).

$$
\eta_{\mathrm{i}} / \phi_{\mathrm{ex}}=\eta_{\mathrm{th}} \eta_{\mathrm{glh}} /\left(1-\eta_{\mathrm{th}} \eta_{\mathrm{glh}}\right)
$$

This equation shows that the $\eta_{\mathrm{i}} / \phi_{\mathrm{ex}}$ ratio is not influenced by the cooling loss fraction $\phi_{\mathrm{w}}$ but is predominantly determined by the theoretical thermal efficiency $\eta_{\text {th }}$ and the degree of constant volume $\eta_{\mathrm{glh}}$. It is also clear that the combustion efficiency $\eta_{\mathrm{u}}$ does not influence the $\eta_{\mathrm{i}} / \phi_{\mathrm{ex}}$ ratio. Because the theoretical thermal efficiency $\eta_{\mathrm{th}}$, which is mainly determined by the compression ratio, is almost constant for an engine, then, unless the degree of constant volume is lowered, the rate of increase in the indicated thermal efficiency by cooling loss reductions is larger than or equal to that in the exhaust loss fraction.

Figure 3 shows the $\eta_{\mathrm{i}} / \phi_{\mathrm{ex}}$ ratio versus the degree of constant volume $\eta_{\mathrm{glh}}$ with different theoretical thermal efficiencies, $\eta_{\mathrm{th}}$. For each value of theoretical thermal efficiency $\eta_{\mathrm{th}}$, the $\eta_{\mathrm{i}} / \phi_{\mathrm{ex}}$ ratio decreases with the degree of 
constant volume $\eta_{\mathrm{glh}}$. With a theoretical thermal efficiency $\eta_{\text {th }}$ of 0.575 , which is for the compression ratio of $\varepsilon=8.5$ and the specific heat ratio of $\gamma=1.4$, the values of the degree of constant volume $\eta_{\text {glh }}$ below 0.87 will cause the exhaust loss fraction $\phi_{\mathrm{ex}}$ to exceed the indicated thermal efficiency $\eta_{\mathrm{i}}$. The critical value of the degree of constant volume $\eta_{\mathrm{glh}}$, which results in $\eta_{\mathrm{i}} / \phi_{\mathrm{ex}}=1$, increases with decreases in the theoretical thermal efficiency $\eta_{\mathrm{th}}$ and compression ratio $\varepsilon$. Lower compression ratios need higher degrees of constant volume to improve the thermal efficiency by cooling loss reductions. Therefore, a method that reduces cooling loss without lowering the degree of constant volume will be effective to improve the thermal efficiency of internal combustion engines.

\subsection{Experimental results in a hydrogen combustion engine}

Figure 4 shows the combustion pressure $P$ and apparent rate of heat release $\mathrm{d} Q / \mathrm{d} \theta$ in a spark ignition engine fueled with hydrogen, for different spark ignition timings $\theta_{\text {ig }}$. The data were measured in an engine with the combustion chamber shown in Figure 5. It has a bore of $85 \mathrm{~mm}$, a stroke of $88 \mathrm{~mm}$, and a compression ratio of 8.5 . The engine speed was $n=1500 \mathrm{rpm}$, and a homogenous mixture of hydrogen and air is charged to the intake pipe of the engine. The mixing ratio is set to soichiometric which result in higher cooling losses than leaner mixture conditions. Homogenously charged hydrogen engines may suffer from backfire, flame propagation back to the intake pipe, particularly with stoichiometric mixtures. To avoid backfiring, the volumetric efficiency was controlled at $\eta_{\mathrm{v}}$ $=50 \%$ by a throttle valve. The combustion pressure was measured with a piezoelectric type pressure transducer (AVL GM12D) installed in the cylinder head and averaged over 45 cycles. The apparent rate of heat release $\mathrm{d} Q / \mathrm{d} \theta$ was calculated by Equation (6) with the pressure data.

Figure 4 indicates that the apparent rate of heat release $\mathrm{d} Q / \mathrm{d} \theta$ turns negative just after the end of combustion, and this signifies that a part of heat released by the combustion is transferred to the combustion chamber walls. The efficiency factors derived from the experimental results are shown in Figure 6. A retardation in the spark ignition timing $\theta_{\text {ig }}$ effectively reduces the cooling loss fraction $\phi_{\mathrm{w}}$, however the indicated thermal efficiency $\eta_{\mathrm{i}}$ is not improved when the ignition timing is later than top dead center because of the increase in the exhaust loss fraction $\phi_{\mathrm{ex}}$ due to the decreased degree of constant volume $\eta_{\mathrm{glh}}$. The results for the $\eta_{\mathrm{i}} / \phi_{\mathrm{ex}}$ ratio show that the exhaust loss fraction $\phi_{\text {ex }}$ exceeds the indicated thermal efficiency $\eta_{\mathrm{i}}$ when the degree of constant volume $\eta_{\mathrm{glh}}$ falls below 0.87 . This critical degree of constant volume value obtained from the experiments is exactly the same as that obtained in the calculations in Section 3.1. This agreement suggests the accuracy of the analytical method in this investigation. Figure 7 shows the exhaust gas temperature $T_{\text {ex }}$ and the exhaust loss fraction $\phi_{\mathrm{ex}}$ against the ignition timing $\theta_{\text {ig. }}$. Results with methane at the same experimental conditions are also shown in the figure. The trends in the exhaust loss fraction are similar to those of the exhaust gas temperature, and this suggests the calculation of the exhaust loss fraction using Equation (16) to be qualitatively accurate.

Retardation of the spark ignition timing can decrease the cooling loss by lowering the maximum combustion temperature. However this method also decreases the degree of constant volume. Therefore, a method to reduce the cooling loss without lowering the degree of constant volume is required to improve the thermal efficiency of hydrogen combustion engines.

\subsection{Reduction of cooling loss by direct injection stratified charge}

Figure 8 shows details of the experimental results with direct injection stratified and the homogeneous charge combustion. The data were obtained in the constant volume combustion vessel shown in Figure 9. After introducing a homogeneously premixed lean mixture of hydrogen and air into the vessel, additional hydrogen was directly injected and ignited with a spark discharge. The lean mixture of hydrogen and air were generated in advance in an external mixing chamber shown in Figure 10. The direct injection of hydrogen was achieved by using an electromagnetic fuel injector with a single-hole nozzle. The fuel was fed to the injector at $3 \mathrm{MPa}$. The initial pressure and temperature in the vessel before the fuel injection were atmospheric and room temperature. The spark location was $20 \mathrm{~mm}$ from the tip of the injector on the injection axis. Ignition was at $20 \mathrm{~ms}$ after the end of injection. Pressure data measured with a piezoelectric type pressure transducer (Kistler 7061) were used to calculate the apparent rate of heat release, the cooling loss fraction, and other parameters. The apparent rate of heat release $\mathrm{d} Q / \mathrm{d} t$ in the constant volume combustion vessel was calculated with the following equation.

$$
\mathrm{d} Q / \mathrm{d} t=V /(\gamma-1) \mathrm{d} P / \mathrm{d} t-P V /(\gamma-1)^{2} \mathrm{~d} \gamma / \mathrm{d} t
$$

The instantaneous wall temperature was measured with a thin film type thermocouple (Medtherm TCS-103E, chromel-constantan type). The measured temperature was used to calculate the instantaneous heat flux to the wall. The concentration of oxygen in the burned gas was measured with a TCD analyzer (Hitachi G-5000) and used for 
calculations of the combustion efficiency.

The mixture conditions for the stratified combustion were chosen to achieve a curve of the apparent rate of heat release $\mathrm{d} Q / \mathrm{d} t$ similar to that in the homogeneous stoichiometric combustion. The overall excess air ratio and the directly injected fuel fraction to the total supplied fuel are $\lambda=1.5$ and $20 \%$. The axes of Figure 8 are the pressure $P$, the apparent rate of heat release $\mathrm{d} Q / \mathrm{d} t$, the apparent heat release fraction $Q / Q_{\text {fuel }}$ and the instantaneous heat flux $q$ against the elapsed time after spark ignition. While the curves for the pressure $P$ and the apparent rate of heat release $\mathrm{d} Q / \mathrm{d} t$ indicate a similar strength and duration of combustion for the two cases, the apparent heat release fraction $Q / Q_{\text {fuel }}$ is larger with the stratified combustion; there is no significant difference in the combustion efficiency $\eta_{\mathrm{u}}$ for the two cases. The combustion efficiency $\eta_{\mathrm{u}}$ and Equation (9) show that the increase in the apparent heat release fraction $Q / Q_{\text {fuel }}$ is caused by a lowered cooling loss fraction $\phi_{\mathrm{w}}$. The instantaneous heat flux is clearly decreased by the stratified charge. The decreased heat transfer to the wall can be attributed to a lowered burned gas temperature in the near wall region due to the much leaner mixture formed there by the stratification.

While the indicated thermal efficiency of an internal combustion engine is expressed by Equation (3), the concept of the degree of constant volume cannot be applied to heat releases in a constant volume combustion vessel. However, if the heat release curves for the two cases shown in Figure 8 are obtained in an internal combustion engine, the two cases would have similar values of the degree of constant volume $\eta_{\mathrm{glh}}$. The theoretical thermal efficiency $\eta_{\text {th }}$, which is determined by the compression ratio and the specific heat ratio of the working fluid, remains almost constant for an engine, and the characteristics of the indicated thermal efficiency $\eta_{\text {i }}$ correspond to those of $\eta_{\mathrm{u}}\left(1-\phi_{\mathrm{w}}\right)$ in Equation (3) or $Q / Q_{\text {fuel }}$ in the following equation.

$$
\eta_{\mathrm{i}}=\eta_{\mathrm{th}} \eta_{\mathrm{glh}} Q / Q_{\text {fuel }}
$$

Therefore, the results in Figure 8 indicate that the direct injection stratified charge at specific conditions can decrease the cooling loss fraction without deteriorating the degree of constant volume and so improve the thermal efficiency in hydrogen combustion engines.

Figure 11 shows the calculated diagram factor $\eta_{\mathrm{g}}$ and indicated thermal efficiency $\eta_{\mathrm{i}}$ based on the experimental results for the two cases in Figure 8. The theoretical thermal efficiency was calculated with the compression ratio $\varepsilon$ of 8.5 and the combustion efficiency $\eta_{\mathrm{u}}$ of 0.98 , and a specific heat ratio $\gamma$ of 1.4 for values of the degree of constant volume $\eta_{\mathrm{glh}}$ from 0.8 to 1.0 . Because of the higher diagram factor $\eta_{\mathrm{g}}$ the indicated thermal efficiency $\eta_{\mathrm{i}}$ is about $20 \%$ higher in the direct injection stratified charge than the homogenous charge for each value of the degree of constant volume $\eta_{\mathrm{glh}}$. Figure 12 shows calculated indicated thermal efficiencies $\eta_{\mathrm{i}}$ for different values of the cooling loss fraction $\phi_{\mathrm{w}}$. The cooling loss fraction was set at the values for the stratified charge and the premixed charge obtained from the experimental results in Figure 8. For a feasibility study, cooling loss fractions lower than the experimental values were also employed in the calculations, because actual running engines have higher combustion chamber wall temperatures than the constant volume combustion vessel which is initially at room temperature. For all the cases, the combustion efficiency $\eta_{\mathrm{u}}$, the specific heat ratio $\gamma$, and the degree of constant volume $\eta_{\mathrm{glh}}$ were set at $0.98,1.4$, and 0.95 respectively, reasonable values for hydrogen engines; the compression ratio $\varepsilon$ in the calculations ranges from 8.0 to 18 . The relatively high compression ratios for spark ignition engines were chosen because the direct injection stratified charge generally enables the engines to operate at higher compression ratios [3]. The leaner mixture near walls also suppress knocking which is caused by the auto-ignition of the compressed end mixture and is a major factor limiting the compression ratio in spark-ignition engines, while a higher compression ratio brings higher theoretical efficiency. The results in Figure 12 naturally indicate that the indicated thermal efficiency $\eta_{\mathrm{i}}$ increases with decreasing cooling loss fraction $\phi_{\mathrm{w}}$ and increasing compression ratio $\varepsilon$. With a compression ratio $\varepsilon$ of 14 and the degree of constant volume $\eta_{\mathrm{glh}}$ of 0.95 for example, a $15 \%$ cooling loss fraction achieves a very high value of the indicated thermal efficiency $\eta_{\mathrm{i}}$, above $50 \%$.

In conclusion, a direct injection stratified charge, which reduces the cooling loss without lowering the degree of constant volume, can be effective to improve the thermal efficiency of hydrogen combustion engines.

\section{Conclusion}

This study investigated the relation between cooling loss reductions and thermal efficiency improvements by the direct injection stratified charge in hydrogen combustion engines. The conclusions of the study are as follows:

(1) In internal combustion engines, the rate of increase in indicated thermal efficiency by reducing the cooling loss is not lower than that in the exhaust loss fraction unless the degree of constant volume is decreased. Therefore, a method to reduce cooling loss without deteriorating the degree of constant volume is necessary to improve the thermal efficiency of engines.

(2) When the degree of constant volume is lower than a critical value, the exhaust loss fraction exceeds the indicated thermal efficiency, and the critical degree of constant volume decreases with decrease in the theoretical thermal 
efficiency. Therefore, engines with lower compression ratios require higher degrees of constant volume to improve the thermal efficiency due to the cooling loss reduction.

(3) A retardation in the spark ignition timing can decrease the cooling loss by lowering the maximum combustion temperature. However this method also decreases the degree of constant volume and does not lead to effective thermal efficiency improvements.

(4) A stratified charge by direct fuel injection into a lean mixture reduces the heat transfer from the burning gas to the combustion chamber walls and increases the apparent heat release fraction in hydrogen combustion. The cooling loss reduction can be attributed to a lowered temperature of burned gas adjacent to the combustion chamber walls by the leaner mixture there, formed by the stratification.

(5) The increase in the apparent heat release fraction effectively leads to improvements in thermal efficiency, because the stratified charge reduces the cooling loss without lowering the degree of constant volume. With the compression ratio 14 and the degree of constant volume 0.95 , for example, a $15 \%$ cooling loss fraction achieves the very high value of indicated thermal efficiency, over $50 \%$. A direct injection stratified charge is an effective technique to improve the thermal efficiency of hydrogen combustion engines.

\section{Acknowledgement}

The author expresses gratitude to Ms. Kazu Ando, Mr. Takayuki Futakuchi, Mr. Tomoharu Kuninaga, Mr. Takayuki Hasegawa, and Mr. Keisuke Yamaguchi, former students of Musashi Institute of Technology, for help in the experiments. This study was financially supported in part by the Industrial Technology Research Grant Program from the New Energy and Industrial Technology Development Organization (NEDO) of Japan.

\section{References}

[1] Lewis B, von Elbe G. Combustion, Flames and Explosions of Gases, Academic Press, 1961.

[2] Shudo T, Nabetani S, Nakajima Y. Analysis of the degree of constant volume and cooling loss in a spark ignition engine fuelled with hydrogen. International Journal of Engine Research 2001;2(1):81-92.

[3] Shudo T, Ogawa H, Miyamoto N. The influence of fuel properties on combustion and emissions in a DI stratified charge engine, JSAE Review 1995;16(1):27-33.

[4] Shudo T, Cheng WK, Kuninaga T, Hasegawa T. Reduction of cooling loss by direct injection stratified charge in hydrogen combustion engines. SAE Transactions Journal of Fuels \& Lubricants 2004;112(4):2118-2123.

[5] King RO, Wallace WA, Mahapatra B. The hydrogen engine and the nuclear theory. Canadian Journal of Research $1948 ; 264$.

[6] Stockhausen WF, Natkin RJ, Kabat DM, Reams L, Tang X, Hashemi S, Szwabowski SJ, Zanardelli V. Ford P2000 hydrogen engine design and vehicle development program. SAE Paper 2002; 2002-01-0240.

[7] List H, Thermodynamik der verbrennungskraftmachinen. Springer, 1939.

[8] Shudo T, Nabetani S, Nakajima Y. Influence of specific heats on indicator diagram analysis in a hydrogen-fuelled SI engine. JSAE Review 2001;22(2):224-226.

\section{Nomenclature}

$\begin{array}{ll}\mathrm{n} & \text { Engine speed } \\ \varepsilon & \text { Compression ratio } \\ \mathrm{V}_{\mathrm{h}} & \text { Stroke volume } \\ \mathrm{V}_{\mathrm{c}} & \text { Clearance volume } \\ \eta_{\mathrm{v}} & \text { Volumetric efficiency } \\ \lambda & \text { Excess air ratio } \\ \gamma & \text { Specific heat ratio } \\ \theta & \text { Crank angle } \\ \theta_{\mathrm{ig}} & \text { Spark ignition timing } \\ P & \text { Pressure } \\ V & \text { Volume } \\ \mathrm{d} Q / \mathrm{d} \theta & \text { Apparent rate of heat release } \\ Q & \text { Cumulative apparent heat release } \\ Q_{\text {fuel }} & \text { Supplied fuel heat } \\ Q / Q_{\text {fuel }} & \text { Apparent heat release fraction }\end{array}$


$Q_{\text {B }} \quad$ Cumulative real heat release

$Q_{\mathrm{C}} \quad$ Cumulative cooling loss

$Q_{\text {ex }} \quad$ Exhaust gas heat

$T_{\text {ex }} \quad$ Exhaust gas temperature

$\eta_{\mathrm{i}} \quad$ Indicated thermal efficiency

$\eta_{\text {th }} \quad$ Theoretical thermal efficiency of the Otto cycle

$\eta_{\mathrm{g}} \quad$ Diagram factor

$\eta_{\text {glh }} \quad$ Degree of constant volume

$\eta_{\mathrm{u}} \quad$ Combustion efficiency

$\phi_{\mathrm{w}} \quad$ Cooling loss fraction, $Q_{\mathrm{C}} / Q_{\mathrm{B}}$

$\phi_{\mathrm{ex}} \quad$ Exhaust heat loss fraction, $Q_{\mathrm{ex}} / Q_{\text {fuel }}$

$q \quad$ Heat flux

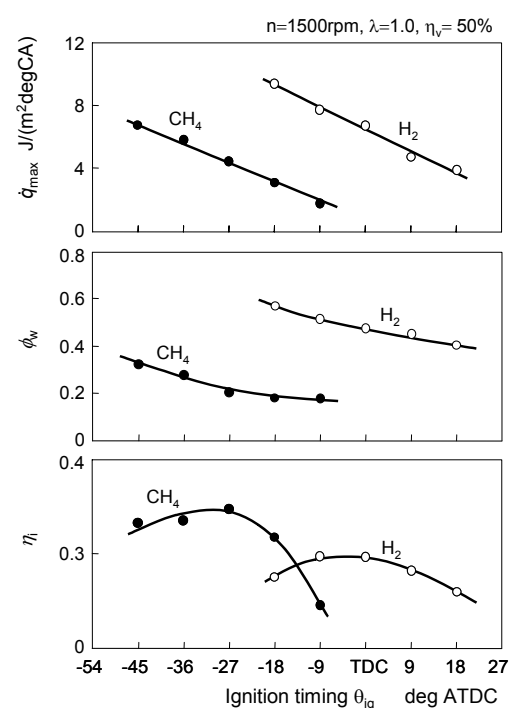

Fig.1 Maximum heat flux to the cylinder head, cooling loss fraction and indicated thermal efficiency for hydrogen and methane combustion.

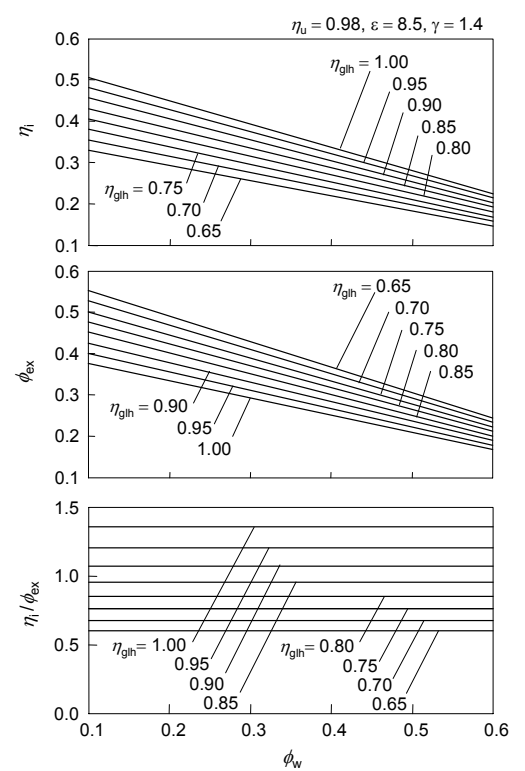

Fig.2 Influence of cooling loss ratio on indicated thermal efficiency and exhaust heat fraction for different degrees of International Association for Hydrogen Energy. Published by Elsevier Ltd. doi: 10.1016/j.jhydene.2007.06.002 
constant volume

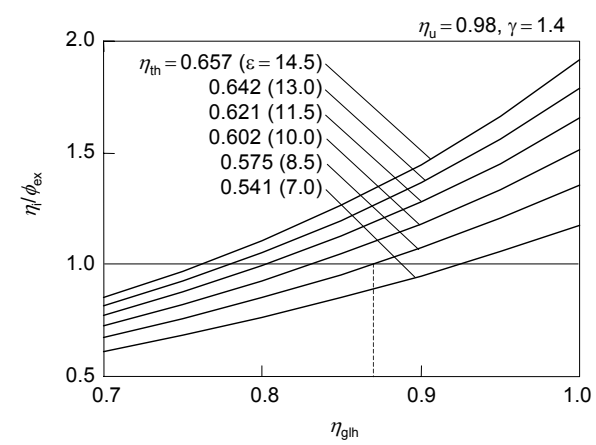

Fig.3 Degree of constant volume versus the fraction of indicated thermal efficiency to exhaust heat fraction for different compression ratios

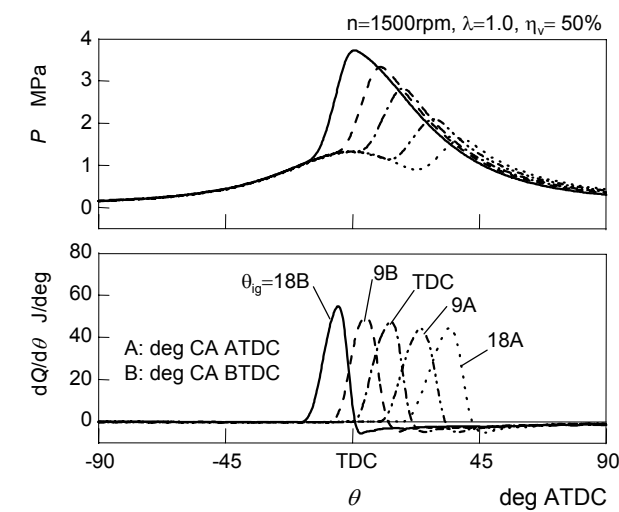

Fig.4 Hydrogen combustion characteristics at different ignition timings

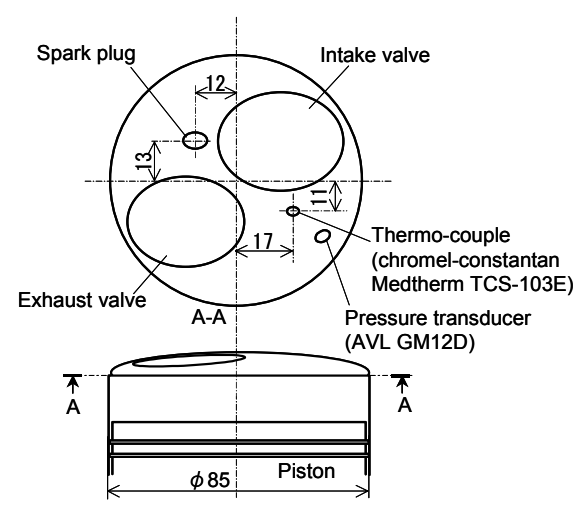

Fig.5 Combustion chamber of the tested engine

International Association for Hydrogen Energy. Published by Elsevier Ltd. doi: 10.1016/j.jhydene.2007.06.002 


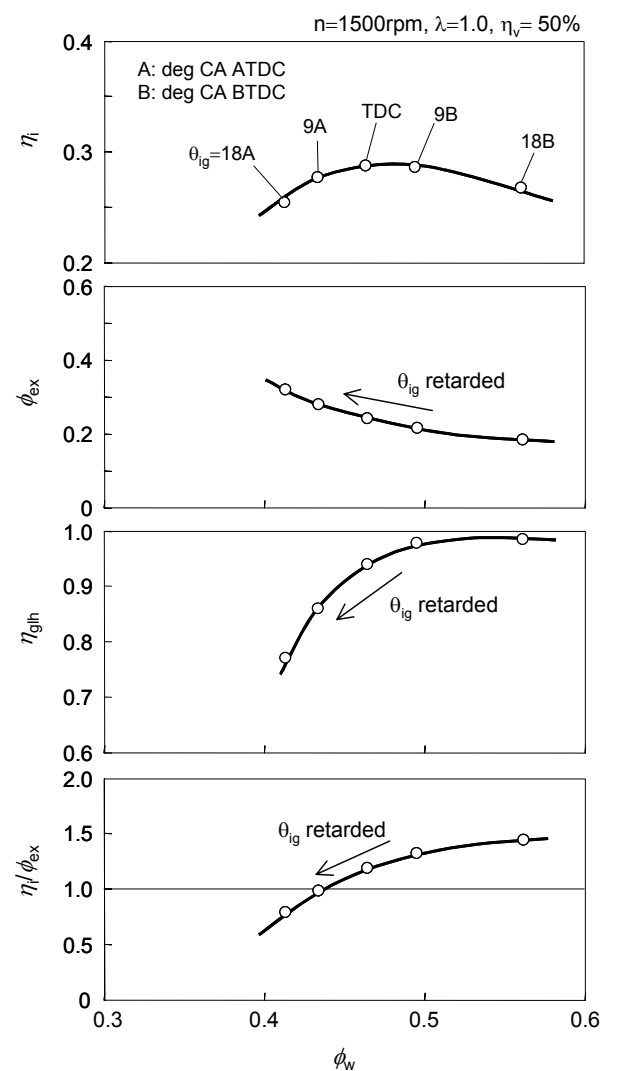

Fig.6 Influence of ignition timing on $\eta_{\mathrm{i}}, \phi_{\mathrm{ex}}, \eta_{\mathrm{glh}}$ and $\eta_{\mathrm{i}} / \phi_{\mathrm{ex}}$ (Curves drawn by hand)

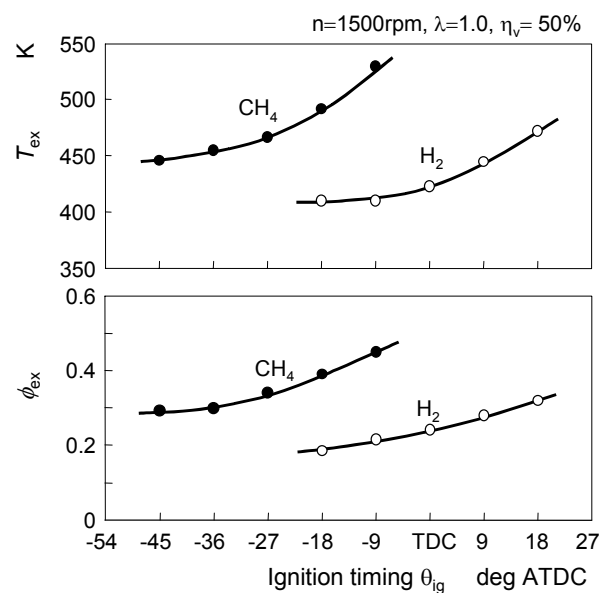

Fig.7 Influence of ignition timing on exhaust gas temperature $\mathrm{T}_{\mathrm{ex}}$ and on $\phi_{\mathrm{ex}}$ (Curves drawn by hand) 


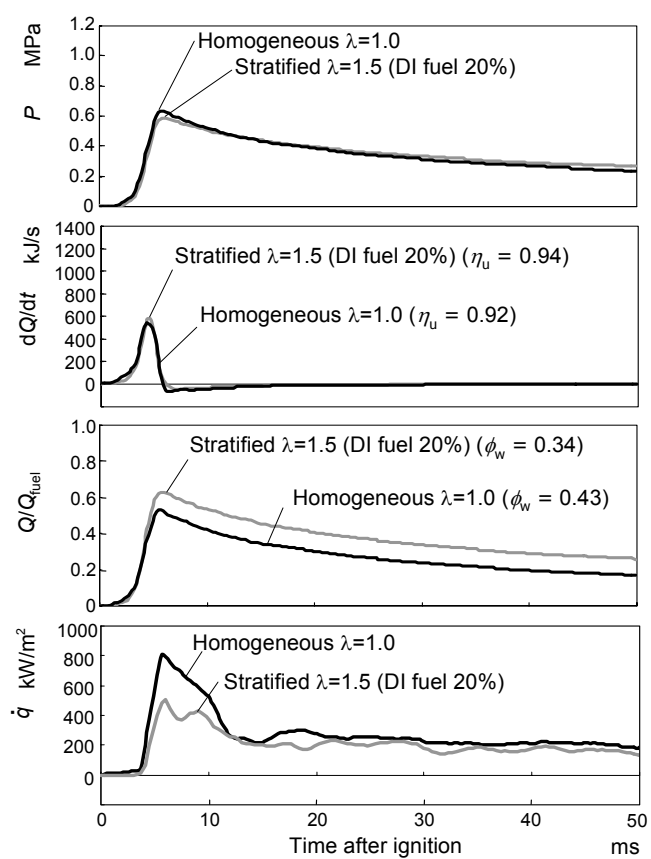

Fig.8 Stratified charge combustion and premixed combustion at similar rates of heat release

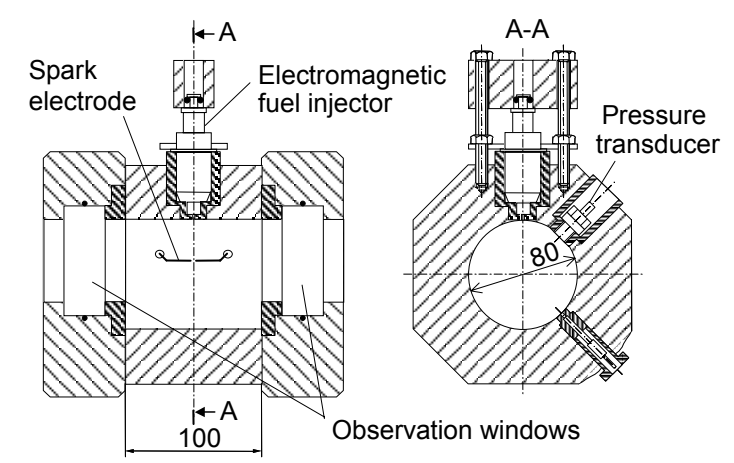

Fig.9 Constant volume combustion vessel used in this investigation

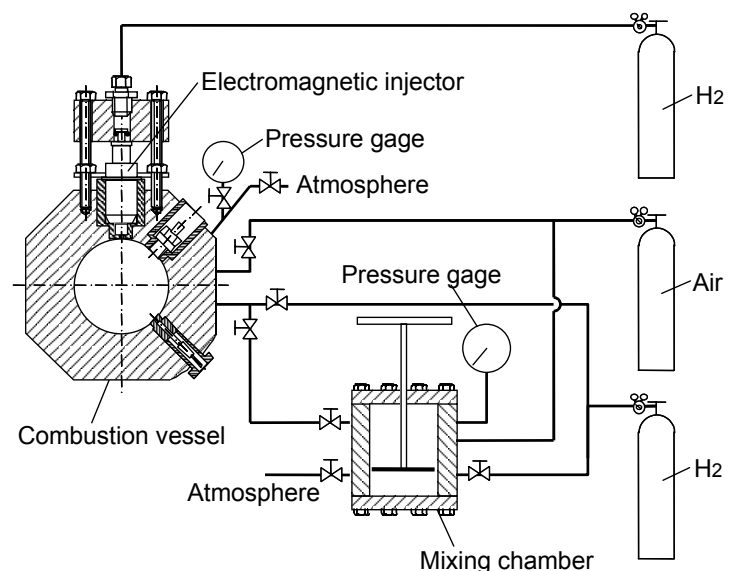

Fig.10 Experimental system for the direct injection stratified charge in the constant volume combustion vessel

International Association for Hydrogen Energy. Published by Elsevier Ltd. doi: 10.1016/j.jhydene.2007.06.002 


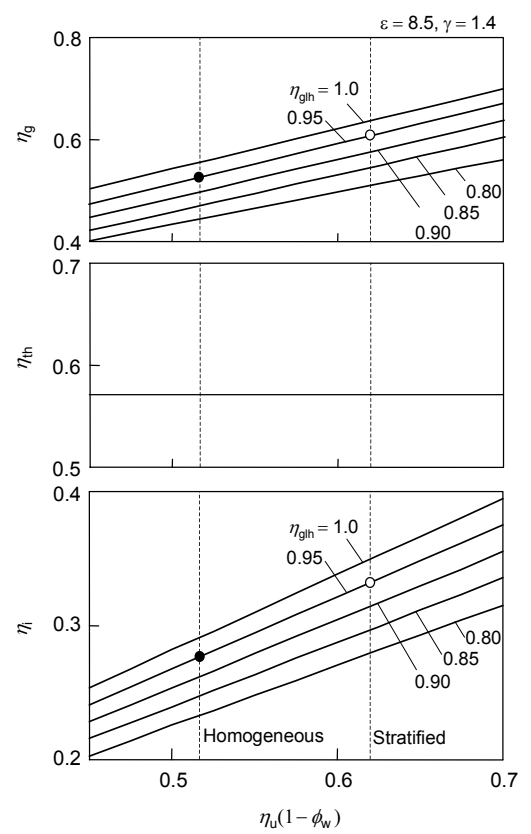

Fig.11 Calculated indicated thermal efficiency based on experimental data in the constant volume combustion chamber

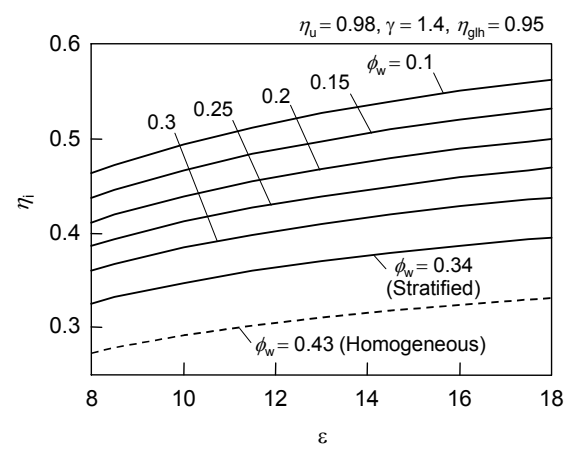

Fig.12 Influence of compression ratio on the calculated indicated thermal efficiency based on data from the constant volume combustion chamber 